\title{
A Cultura Digital em Práticas Educativas: Transformações e Acoplamentos na Formação de Professores de Matemática
}

\author{
La Cultura Digital en Prácticas Educativas: Transformaciones y \\ Acoplamientos en la Formación de Profesores de Matemáticas
The Digital Culture in Educational Practices: Transformations and Couplings in the Training of Math Teachers

Gerson Freitas Luz ${ }^{1}$

Daniele Amaral Fonseca ${ }^{2}$

Daniel da Silva Silveira ${ }^{3}$

Débora Pereira Laurino ${ }^{4}$

\begin{abstract}
Resumo
O operar contínuo da tecnologia digital, em consonância com a globalização econômica, política e social, gera novas formas de comunicação, outras construções culturais e uma diversidade de práticas sociais o que inclui as práticas pedagógicas no âmbito educacional. O objetivo deste trabalho é compreender o operar das tecnologias em uma cultura digital no ensino de Matemática na Educação Básica. Como processo metodológico adotou-se o levantamento bibliográfico em periódicos nacionais da área de Ensino, dos estratos A1, A2 e B1, dos quais surgiram 66 artigos que pautam as práticas pedagógicas em matemática com o uso das tecnologias digitais e a partir da sua leitura e utilizando a Análise de Conteúdo, foi gerada a categoria "Formação de professores de matemática com as tecnologias digitais". A categoria evidencia a importância da formação de professores ser atualizada no modo como se apropria das tecnologias digitais para desenvolver práticas pedagógicas que potencializem a interação e os processos de ensinar e aprender matemática. Destarte, este estudo permitiu concluir que a apropriação das tecnologias digitais pode produzir transformações e acoplamentos na formação dos professores, o que implica na combinação de vários modos de comunicação e de interação, em diferentes níveis de complexidade, de interatividade, de práticas, de modos de pensamento, que levam a ensinar os conceitos matemáticos pelo digital e que pelo seu uso recorrente é gerada uma cultura digital.
\end{abstract}

Palavras-Chave: Cultura Digital; Ensino de Matemática; Formação de Professores.

\section{Resumen}

El funcionamiento continuo de la tecnología digital, en consonancia con la globalización económica, política y social, genera nuevas formas de comunicación, otras construcciones culturales y una diversidad de prácticas sociales lo que incluye las prácticas pedagógicas en el ámbito educativo. El objetivo de este trabajo es comprender el funcionamiento de las tecnologías en una cultura digital en la enseñanza de Matemáticas en la Educación Básica. Como proceso metodológico se adoptó el levantamiento bibliográfico en periódicos nacionales del área de Enseñanza, de los estratos A1, A2 y B1, de los cuales surgieron 66 artículos que pautan las prácticas pedagógicas en matemáticas con el uso de las tecnologías digitales ya partir de su lectura y utilizando el Análisis de Contenido, se generó la categoría "Formación de profesores de matemáticas con las

\footnotetext{
1 Acadêmico do Curso de Licenciatura em Matemática; Universidade Federal do Rio Grande - FURG; Rio Grande, Rio Grande do Sul, Brasil; gersonluz2000@gmail.com

${ }^{2}$ Licenciada em Matemática; Universidade Federal do Rio Grande - FURG; Rio Grande, Rio Grande do Sul, Brasil; danieleamaral4@gmail.com

${ }^{3}$ Doutor em Educação em Ciências; Universidade Federal do Rio Grande - FURG; Rio Grande, Rio Grande do Sul, Brasil; dssilveira@ furg.br

${ }^{4}$ Doutora em Informática na Educação; Universidade Federal do Rio Grande - FURG; Rio Grande, Rio Grande do Sul, Brasil; deboralaurino@furg.br
} 
tecnologías digitales". La categoría evidencia la importancia de la formación de profesores para ser actualizada en el modo en que se apropia de las tecnologías digitales para desarrollar prácticas pedagógicas que potencien la interacción y los procesos de enseñanza y aprendizaje matemática. De este modo, este estudio permitió concluir que la apropiación de las tecnologías digitales puede producir transformaciones y acoplamientos en la formación de los profesores, lo que implica la combinación de varios modos de comunicación y de interacción, en diferentes niveles de complejidad, de interactividad, de prácticas, de modos de pensamiento, que llevan a enseñar los conceptos matemáticos por lo digital y que por su uso recurrente se genera una cultura digital.

Palabras claves: Cultura digital; Enseñanza de Matemática; Formación de profesores.

\begin{abstract}
The continuous operation of digital technology, in accordance with economic, political and social globalization, generates new forms of communication, other cultural constructions and a diversity of social practices which includes the pedagogical practices in the educational scope. The objective of this work is to understand the operation of technologies in a digital culture in the teaching of Mathematics in Basic Education. As a methodological process, a bibliographical survey was adopted in national journals in the Teaching area of the strata A1, A2 and B1, from which 66 articles emerged listing the pedagogical practices in mathematics with the use of digital technologies and from their reading using Content Analysis, the category "Training of teachers of mathematics with digital technologies" was generated. The category highlights the importance of teacher training to be updated in the way it appropriates digital technologies to develop pedagogical practices that enhance interaction and the processes of teaching and learning mathematics.Thus, this study allowed to conclude that the appropriation of digital technologies can produce transformations and couplings in teachers training, which implies the combination of several modes of communication and interaction, in different levels of complexity, interactivity, practices, modes of thought, which lead to teaching mathematical concepts digitally and that by its recurrent use is generated a digital culture.
\end{abstract}

Keywords: Digital Culture; Mathematics Teaching; Teacher training.

\title{
1. Introdução
}

Com o avanço das tecnologias digitais diversas são as pesquisas no Brasil acerca das suas potencialidades nos processos de ensinar e de aprender na formação inicial e continuada de professores (MENDES, 2009; PRETTO, 2011; SILVEIRA, 2017). Entende-se que educar em uma sociedade tecnológica é mais do que instrumentalizar os indivíduos para o uso das tecnologias digitais, é possibilitar que operem os artefatos tecnológicos gerando interação em uma rede dinâmica de aprendizagem fundada no digital.

Entende-se o operar, com base em Maturana e Varela (2001), como um processo que gera uma conduta, um modo de viver, agir e compreender o que se faz. Neste trabalho, o operar da tecnologia digital é concebido como uma forma de significar e compreender os processos de ensinar e de aprender Matemática na Educação Básica a partir do potencial de interação entre sujeitos, bem como uma mecanismo que transforma ou constitui diferentes culturas digitais, que podem ser coerentes com os modos de compreensão e significação dos sujeitos.

Os processos educativos quando pensados, implementados e mediados com o uso de tecnologias digitais podem promover a construção do conhecimento de maneira interativa e 
tendo a interação como um dos elementos essenciais na geração da aprendizagem. Mediar o processo de ensinar e de aprender como uma proposta pedagógica que leve em conta o uso de tecnologias digitais (aplicativos, softwares, ambientes virtuais de aprendizagem, simuladores, planilhas eletrônicas, entre outros), requer dos indivíduos novas formas de considerar o saber humano, de aprender a aprender em um espaço digital, bem como outras maneiras de se apropriar dos saberes científicos. Para tanto, além dos professores apresentarem o conhecimento de como utilizar estas tecnologias digitais nos processos pedagógicos é fundamental conhecer o contexto de seus estudantes para pensar para que e com quem utilizálas no intuito de construir aprendizagens.

Especificamente no ensino de Matemática, quando o professor reflete sobre como vai operar as tecnologias digitais e para quem vai ensinar determinado conceito, pode garantir não somente que o estudante visualize o passo a passo de uma solução por um aplicativo, mas também pode contribuir para compreensão do desenho de uma curva ao plotar em um software, em se tratando da geometria ou de um gráfico de funções, e ainda simular diferentes comportamentos a partir da variação de parâmetros de um problema. Por isso, acredita-se que as tecnologias digitais quando operadas com finalidade pedagógica podem transformar professores e estudantes em produtores de culturas e conhecimentos, superando a lógica de serem recursos somente para o consumo da informação.

A partir deste contexto, o presente trabalho busca compreender o operar das tecnologias em uma cultura digital no ensino de Matemática na Educação Básica. Para compreender o fenômeno investigado adotou-se como procedimento metodológico o levantamento bibliográfico em periódicos nacionais da área de Ensino o qual será descrito na próxima seção.

\section{Processo metodológico}

O processo metodológico deste trabalho parte do levantamento bibliográfico em periódicos nacionais para compreender o operar das tecnologias digitais no ensino de Matemática na Educação Básica. Para isso, foi realizada uma busca em periódicos científicos com base na classificação do Qualis de 2013 a 2016 da Coordenação de Aperfeiçoamento de Pessoal de Nível Superior (CAPES), disponível na Plataforma Sucupira, na área de Ensino, e que apresentavam no seu título a palavra "tecnologia" ou "matemática". Assim, dentre os periódicos dos estratos A1, A2 e B1, surgiram 28. Destes foram selecionados 17 em que foi constatado, a partir da leitura de seus editoriais, artigos que pautam as práticas educativas em matemática com o uso das tecnologias digitais. 
Em consulta aos 17 periódicos, utilizou-se como chaves de pesquisa para seleção dos artigos as palavras "tecnologias digitais" e "matemática". Nessa primeira etapa de seleção, foi registrado, em 194 artigos, a discussão sobre o uso de tecnologias digitais no ensino da Matemática. Na segunda etapa, foi selecionado 66 artigos, por considerar como critério a temática das tecnologias digitais no ensino da Matemática na Educação Básica. Cabe salientar que se demarcou como critério temporal os artigos publicados a partir do ano de 2004, por este ser o ano do surgimento da Web 2.0, ou seja, período de grande expansão das tecnologias digitais.

Com base na leitura dos 66 artigos selecionados e utilizando a Análise de Conteúdo (BARDIN, 2011), foram geradas duas categorias as quais se intitulou de "Formação de professores de matemática com as tecnologias digitais" e "Os processos de ensinar e de aprender matemática no operar das tecnologias digitais". Para este trabalho irá se discorrer sobre a primeira categoria que foi gerada pela análise de 15 artigos mapeados os quais são apresentados a seguir na Tabela 1 .

Tabela 1 - Artigos que compõem a primeira categoria de análise.

\begin{tabular}{|c|c|c|c|}
\hline Periódico & Código & Título & Ano \\
\hline \multirow[t]{2}{*}{ Bolema } & $\mathrm{T} 1$ & $\begin{array}{l}\text { O uso das TIC nas práticas dos professores de } \\
\text { matemática da rede básica de ensino: o projeto } \\
\text { mapeamento e seus desdobramentos }\end{array}$ & 2015 \\
\hline & $\mathrm{T} 2$ & $\begin{array}{l}\text { Identificação e análise das dimensões que } \\
\text { permeiam a utilização das tecnologias de } \\
\text { informação e comunicação nas aulas de } \\
\text { matemática no contexto da formação de } \\
\text { professores }\end{array}$ & 2006 \\
\hline \multirow[t]{2}{*}{$\begin{array}{c}\text { Educação Matemática em } \\
\text { Revista }\end{array}$} & $\mathrm{T} 3$ & $\begin{array}{l}\text { Luz, câmera, ação... quando professores que } \\
\text { ensinam matemática nos anos iniciais criam } \\
\text { filmes de curta-metragem }\end{array}$ & 2017 \\
\hline & T4 & Tecnologias digitais e educação matemática & 2013 \\
\hline SABEM-RS & T5 & $\begin{array}{c}\text { Possibilidades e limitações da inserção de } \\
\text { tecnologias nas aulas de matemática no ensino } \\
\text { fundamental }\end{array}$ & 2012 \\
\hline $\begin{array}{c}\text { Educação Matemática } \\
\text { Pesquisa }\end{array}$ & T6 & $\begin{array}{l}\text { A contextualização e os objetivos digitais de } \\
\text { aprendizagem na educação básica: o currículo e } \\
\text { a sua aplicação na matemática }\end{array}$ & 2016 \\
\hline
\end{tabular}


RELACult - Revista Latino-Americana de Estudos em Cultura e Sociedade

Revista Latinoamericana de Estudios en Cultura y Sociedad | Latin American Journal of Studies in Culture and Society V. 05, ed. especial, abr., 2019, artigo n ${ }^{\circ} 1188$ | claec.org/relacult | e-ISSN: 2525-7870

\begin{tabular}{|c|c|c|c|}
\hline & $\mathrm{T} 7$ & $\begin{array}{c}\text { Desenvolvimento do pensamento estatístico } \\
\text { como suporte computacional }\end{array}$ & 2015 \\
\hline $\begin{array}{l}\text { Jornal Internacional de } \\
\text { Estudos em Educação } \\
\text { Matemática }\end{array}$ & T8 & $\begin{array}{c}\text { Tecnologia e aprendizagem de conceitos } \\
\text { matemáticos }\end{array}$ & 2017 \\
\hline $\begin{array}{l}\text { Ensino de Ciência e } \\
\text { Matemática }\end{array}$ & T9 & $\begin{array}{c}\text { Produzindo problemas abertos utilizando } \\
\text { tecnologias digitais no processo de formação } \\
\text { inicial de professores de matemática }\end{array}$ & 2017 \\
\hline \#tear & T10 & $\begin{array}{l}\text { Piaget e Vygotsky: um paralelo entre as ideias } \\
\text { de cooperação e interação no desenvolvimento } \\
\text { de um espaço de aprendizagem digital }\end{array}$ & 2013 \\
\hline \multirow[t]{2}{*}{ Renote } & T11 & $\begin{array}{l}\text { O uso das tecnologias digitais para o ensino de } \\
\text { matemática financeira: uma revisão sistemática } \\
\text { de literatura }\end{array}$ & 2016 \\
\hline & T12 & $\begin{array}{l}\text { Pensar-com tecnologias digitais de matemática } \\
\text { dinâmica }\end{array}$ & 2015 \\
\hline $\begin{array}{c}\text { Revista Educação \& } \\
\text { Tecnologia }\end{array}$ & T13 & $\begin{array}{l}\text { A formação de professores do magistério e as } \\
\text { possibilidades de uso do celular no ensino de } \\
\text { matemática para uma alimentação saudável }\end{array}$ & 2016 \\
\hline $\begin{array}{l}\text { Revista Perspectivas da } \\
\text { Educação Matemática }\end{array}$ & T14 & $\begin{array}{l}\text { A integração das tecnologias digitais ao ensino } \\
\text { de matemática: desafio constante no cotidiano } \\
\text { escolar do professor }\end{array}$ & 2015 \\
\hline Em TEIA & T15 & $\begin{array}{c}\text { Modelagem matemática na inserção de } \\
\text { tecnologias da informação para o ensino de } \\
\text { geometria analítica }\end{array}$ & 2013 \\
\hline
\end{tabular}

Fonte: os autores (2018)

As discussões acerca do operar das tecnologias digitais no ensino de Matemática na Educação Básica, presentes nos artigos acima mapeados, contribuem para a disseminação de pesquisas sobre a cultura digital na formação e na ação de professores de Matemática, bem como ajudam a compreender o que outros autores já realizaram e problematizaram sobre o assunto. Nesse sentido, na próxima seção, se fará menção a alguns dos artigos analisados que discorrem sobre a utilização das tecnologias digitais na docência em matemática.

\section{Transformações e acoplamentos na formação de professores de Matemática}


Ao analisar os artigos que geraram a categoria "Formação de professores de matemática com as tecnologias digitais", percebeu-se que a apropriação dos artefatos tecnológicos requer atenção e olhar crítico sobre a intencionalidade pedagógica para uma utilização contextualizada da ferramenta, bem como pode modificar a cultura alterando as formas de se atuar em sociedade, isso porque requer a aquisição e a compreensão de simbologias, a formalização do conhecimento e de representações que utilizam equipamentos computacionais e dispositivos móveis.

De acordo com Costa (2008), a cultura digital é compreendida como processos, experiências, vivências e escolhas que ocorrem frente ao excesso de informações e serviços que circulam pelos bancos de dados, redes e dispositivos digitais. Para Lemos e Lévy (2010), a cultura digital é uma forma sociocultural que possibilita a mudança nos hábitos sociais, nas práticas de consumo e produção cultural, o que potencializa novas relações de trabalho e de lazer, outras configurações na sociabilidade e na comunicação social pelo operar das tecnologias digitais.

O campo educacional, como parte do sistema social, é influenciado e influencia práticas com o uso de tecnologias, por meio da comunicação entre pessoas e no acesso a espaços culturais divulgados no digital, que refletem diretamente na produção do conhecimento e nos relacionamentos interpessoais, revelando uma cultura em que os meios e as tecnologias digitais vão se tornando inerentes ao viver das pessoas. No entanto, mesmo estes artefatos imbricados ao cotidiano de muitas pessoas é apontado no artigo T2 que existem desafios em relação a implementação e disseminação das tecnologias digitais na formação e na ação de professores, o que reflete nas práticas de ensino na escola.

Os sujeitos que constituem a comunidade escolar (professores, estudantes, pais e gestores) precisam se envolver e valorizar projetos pedagógicos que utilizam as tecnologias digitais como ferramenta na produção do conhecimento, pois uma prática pedagógica mediada por estas ferramentas pode contribuir para a cognição humana. Neste contexto, a cognição humana é interpelada pelos diferentes meios de comunicação online, devido às possibilidades de interação que ocorrem entre os sujeitos nestes espaços. Os dispositivos digitais influenciam no processo do pensamento e, consequentemente, do aprender. Segundo Maturana (1993), a aprendizagem é uma transformação estrutural da convivência, e nesse processo de transformação a interação é um dos mecanismos operacionais da aprendizagem.

Para Silva (2015, p.43), a interação é compreendida como "as ações mútuas entre pessoas, entre pessoas e coisas ou somente entre coisas". Assim, como seres humanos, vive-se na recorrência de emoções e ações que se entrelaçam formando redes consensuais. As redes 
são consensuais, pois o consenso é uma coordenação de ação como resultado da conversação que se estabelece por meio de interações recorrentes (MATURANA, 2014). Dessa forma, os domínios de ações que envolvem a cultura e a sociedade, são constituídos em diferentes redes de conversação através da recorrência de interações dos sujeitos.

A importância da interação entre os sujeitos é cada vez mais destacada no processo de ensinar com as tecnologias digitais. Para Porto (2003) e Kenski (2007), as tecnologias digitais no espaço educativo precisam extrapolar a ideia de ferramentas de auxílio ao processo de ensinar, o que significa considerar o como se opera as tecnologias digitais a fim de possibilitar a diferença na forma de se ensinar os conteúdos e potencializar um aprender significativo do sujeito. Por exemplo, ao operar uma tecnologia digital como o Winplot (software gratuito e interativo que apresenta interface algébrica e gráfica), para discutir o conceito de funções e suas propriedades, o importante é saber se o estudante já apresenta um saber sobre o assunto, e se na interação com esse software e com os outros colegas, que estão em uma dinâmica colaborativa de interações, realizou coordenações que o levaram a novas relações e consequentemente a outras aprendizagens.

Porém, o artigo T1 evidencia a existência de uma cultura de ensino da matemática ainda centrada na fragmentação do conhecimento e desvinculada do contexto dos estudantes. Acredita-se que uma maneira de modificar essa realidade é migrando o uso de tecnologias digitais para um ambiente educativo que integre o desenvolvimento de temas relacionados à diversas áreas do conhecimento, que considere o trabalho coletivo, a produção, a afetividade, o social e as especificidades de cada estudante. Para tanto, compreende-se que a tecnologia digital quando incorporada na formação de professores não como algo a ser usada eventualmente, mas como um recurso que possibilite a integração entre docentes e estudantes e que leve ao raciocínio lógico, a criação, a criatividade e ao diálogo, pode contribuir para a construção do conhecimento matemático e na constituição como sujeitos em uma cultura digital.

Por isso, o artigo T9 discute que os formadores de professores ao ser atualizarem no modo como se apropriam das tecnologias digitais podem desenvolver práticas pedagógicas que potencializem a interação e os processos de ensinar e aprender matemática. Além disso, este artigo aborda a importância de se criar e/ou estabelecer trocas colaborativas entre seus pares, pois o diálogo e a socialização de experiências levam a reflexão da atividade educativa o que para Perez (2004, p. 252), "é vista como um processo em que o professor analisa sua prática, compila dados, descreve situações, elabora teorias, implementa e avalia projetos e partilha suas ideias com colegas e alunos, estimulando discussões em grupos", bem como 
contempla conhecimentos operacionais como também saberes pedagógicos que podem auxiliar na compreensão, na autonomia e na análise de situações em sala de aula.

O artigo T9 ressalta também a importância da resolução de problemas ser desenvolvida por meio das tecnologias digitais uma vez que possibilitam a captura e reprodução de procedimentos realizados, um reolhar e, portanto, a reflexão de um processo; além da agilidade e da diversidade de formas de compartilhar os procedimentos. Operar softwares dinâmicos como, por exemplo, o GeoGebra (software que permite trabalhar conceitos de geometria e álgebra em uma interface gráfica), gera possibilidades diversificadas, que proporciona interatividade entre os estudantes, a tecnologia digital e o conceito abordado, concepção esta também encontrada no artigo T12.

Para Brito e Almeida (2005), o uso de tecnologias digitais auxilia os estudantes em trabalhos, que muitas vezes são árduos, minimizando esforços, como é o caso na determinação de parâmetros de uma função por meio de um conjunto de informações. Esse fato permite que os estudantes tenham a oportunidade de concentrar seus esforços na interpretação e na análise das situações que envolvem o problema, assim como simular diferentes condições para enriquecer a sua análise.

$\mathrm{O}$ artigo T13 comenta que mesmo a escola tendo um laboratório de informática o seu uso não é recorrente, pois ainda há carência em explorar o real potencial das ferramentas tecnológicas e entrelaçar o seu operar com os objetivos e os processos pedagógicos. Isso remete pensar que falta na formação de professores ainda a discussão e experienciação de práticas educativas recorrentes que levem a construção de saberes pedagógicos atrelados ao uso das tecnologias digitais. Nesse sentido, é apontado por Pereira, Silva e Novello (2018) a necessidade do oferecimento de cursos de formação inicial e continuada que tenham as tecnologias digitais imbricadas no fazer educativo, contemplando não somente aspectos técnicos, mas em especial os pedagógicos na intenção de articular os conteúdos conceituais aos procedimentais e atitudinais.

A finalidade pedagógica do artefato tecnológico precisa ir ao encontro dos objetivos da atividade a ser desenvolvida, bem como ser analisada a fim de proporcionar a investigação e o desafio do intelecto dos estudantes. Marques (2003) salienta que a utilização de recursos didáticos em sala de aula não garante uma aprendizagem, pois não são os objetos e equipamentos que fazem a diferença, mas sim o modo como são operados. Assim, é o uso didático-pedagógico deles que os faz instrumentos do processo de ensinar e de aprender, o que pode implicar na ressignificação do método único de se ensinar. 
Nesta perspectiva, o operar das tecnologias digitais não é apenas destinada aos estudantes, mas também aos professores e outros sujeitos que se envolvem no processo de construção do conhecimento. A utilização da tecnologia digital no processo de ensinar e de aprender desafia a crença e a postura dos professores em relação à construção de uma nova identidade docente, principalmente, em relação ao valor que agregam ao ensino.

Criar espaços de formação permanente em que os professores tenham a oportunidade de vivenciar a experiência no operar das tecnologias digitais, pode possibilitar a problematização do uso dessas ferramentas na sala de aula. Desta forma, é possível integrá-las e imbricá-las ao planejamento de aula, com vistas à significação dos conceitos vinculados à sua disciplina e à formação dos sujeitos.

\section{Considerações finais}

Neste trabalho buscou-se constituir elementos que permitiu compreender o operar das tecnologias digitais no ensino de Matemática na Educação Básica por meio do mapeamento e análise de artigos científicos. Acredita-se que a apropriação das tecnologias digitais pode produzir transformações e acoplamentos na formação dos professores, pois a combinação de vários modos de comunicação e de interação, em diferentes níveis de complexidade, de interatividade, de práticas, de modos de pensamento, levam a ensinar os conceitos matemáticos pelo digital e que pelo seu uso recorrente é gerada uma cultura digital.

É complexo caracterizar uma formação de professores ideal, pelo fato de se ter uma diversidade de objetivos, interesses, sujeitos e contextos. Desse modo, mais do que a necessidade de se estabelecer fronteiras entre a forma de se utilizar as tecnologias digitais, pode-se pensar que é no refletir sobre os processos e ações que potencializam a apropriação tecnológica dos professores e dos estudantes, é preciso considerar o engendramento de uma prática que inclua os saberes pedagógicos, conceituais, tecnológicos ou contextuais. Assim, a relevância da prática pedagógica estará centrada na mobilização de saberes que ela possibilita ao realizá-la o que poderá gerar uma mudança tanto na formação de professores de matemática como na atuação dos docentes na Educação Básica.

Através desta pesquisa destaca-se a necessidade de aprofundar os estudos sobre a cultura digital no ensino de Matemática, tendo em vista que contribuem para a disseminação de pesquisas sobre o operar das tecnologias digitais na escola, e que possam gerar transformações e acoplamentos no papel do professor, provocando-o a refletir sobre sua prática, o que pode mudar a dinâmica da sala de aula e, consequentemente, a cultura de ensino. 


\section{Referências}

BARDIN, L. Análise de conteúdo. São Paulo: Edições 70, 2011. 280 p.

BRITO, D. S.; ALMEIDA, L. M. W. O conceito de função em situações de modelagem matemática. Revista Zetetiké, v. 13, n. 23, p. 63-86, 2005. ISSN 2176-1744. Disponível em: https://periodicos.sbu.unicamp.br/ojs/index.php/zetetike/article/view/8646980. Acesso em: 09 set. 2018.

COSTA, R. A cultura digital. São Paulo: Publifolha, 2008. 87 p.

KENSKI, V. M. Educação e tecnologias: o novo ritmo da informação. Campinas: Papirus, 2007. 144 p.

LEMOS, A.; LÉVY, P. O futuro da internet: em direção a uma ciberdemocracia planetária. São Paulo: Paulus, 2010. 264 p.

MARQUES, M. O. Formação do profissional da educação. Ijuí: Editora Unijuí, 2003. 220 p.

MATURANA, H. Cognição, ciência e vida cotidiana. Belo Horizonte: Editora UFMG, 2014. 203 p.

MATURANA, H. Uma nova concepção de aprendizagem. Revista Dois Pontos, v. 2, n. 15, p. 28-35, 1993. Disponível em: https://philpapers.org/rec/MATUNC. Acesso em: 11 out. 2018.

MATURANA, H.; VARELA, F. A árvore do conhecimento: as bases biológicas da compreensão humana. São Paulo: Palas Athena, 2001. 288 p.

MENDES, I. A. Matemática e investigação em sala de aula. Tecendo redes cognitivas na aprendizagem. São Paulo: Livraria da Física, 2009. 215 p.

PEREIRA, F. D.; SILVA, L. M. S.; NOVELLO, T. P. A relação entre a formação docente e o tecnostress. RELACult - Revista Latino-Americana de Estudos em Cultura e Sociedade, v. 4, ed. especial, p. 01-10, fev. 2018. ISSN 2525-7870. Disponível em: http://periodicos.claec.org/index.php/relacult/article/view/721/386. Acesso em: 21 dez. 2018.

PEREZ, G. Prática reflexiva do professor de matemática. In: BICUDO, M. A. V. (Org.). Educação Matemática: pesquisa em movimento. São Paulo: Cortez, 2012. p. 250-263.

PORTO, T. M. E. As mídias e os processos comunicacionais na formação docente na escola. In: PORTO, T. M. E. (Org.). Redes em construção: meios de comunicação e práticas educativas. Araraquara: JM, 2003. p. 79-110.

PRETTO, N. L. O desafio de educar na era digital: educações. Revista Portuguesa de Educação, v. 24, n. 1, p. 95-118, 2011. ISSN: 0871-9187. Disponível em: https://revistas.rcaap.pt/rpe/article/view/3042/2459. Acesso em: 02 dez. 2018. 
SILVA, M. Interação e interatividade: sugestões para docência na cibercultura. In: PORTO, C. et al. (Orgs.). Pesquisa e mobilidade na cibercultura: itinerâncias docentes. Salvador: Edufba, 2015. p. 43-64.

SILVEIRA, D. S. Redes de conversação em uma cultura digital: um modo de pensar, agir e compreender o ensino de Matemática na educação superior. 2017. 162 f. Tese (Doutorado em Educação em Ciências: Química da Vida e Saúde), Universidade Federal do Rio Grande - FURG, Rio Grande. 2017. 